\title{
Increasing the Size of a Piece of Popcorn
}

\author{
Paul V. Quinn Sr. \\ Department of Physical Sciences, Kutztown University, Kutztown, Pennsylvania \\ 19530 \\ Daniel C. Hong \\ Physics, Lewis Laboratory, Lehigh University, Bethlehem, Pennsylvania 18015 \\ J. A. Both \\ Department of Radiation Oncology, Stanford School of Medicine, 300 Pasteur Drive, \\ Stanford, California 94305
}

\begin{abstract}
Popcorn is an extremely popular snack food in the world today. Thermodynamics can be used to analyze how popcorn is produced. By treating the popping mechanism of the corn as a thermodynamic expansion, a method of increasing the volume or size of a kernel of popcorn can be studied. By lowering the pressure surrounding the unpopped kernel, one can use a thermodynamic argument to show that the expanded volume of the kernel when it pops must increase. In this project, a variety of experiments are run to test the validity of this theory. The results show that there is a significant increase in the average kernel size when the pressure of the surroundings is reduced.
\end{abstract}

\section{Introduction}

Popcorn is an extremely popular snack food, found in many cultures throughout the world. It was first grown by the American Indians, before Columbus discovered America. The mass production of popcorn has become a thriving industry in our society today. Because of the desire to maximize profits, the food industry may have a vested interest in trying to increase the size of a piece of popcorn. Larger popcorn could result in costs savings for mass production as well as creating a snack that is more esthetically pleasing to the consumer. Therefore, larger popcorn would be profitable to the manufacturers as well as more enjoyable for the consumer. Three major parameters of interest to industry are the expansion volume, the flake size, and the percent of unpopped kernels. We respectively assign the following variables to these three parameters with their proper definitions $[1,2]$ :

$$
\begin{aligned}
\sigma & =\frac{\text { total popped volume }\left(\mathrm{cm}^{3}\right)}{\text { original sample weight }(\mathrm{g})}, \\
\pi & =\frac{\text { total popped volume }\left(\mathrm{cm}^{3}\right)}{\text { number of popped kernels }},
\end{aligned}
$$




$$
\omega=\frac{\text { number of unpopped kernels }}{\text { original number of kernels }} \text {. }
$$

In popcorn production, the goal is to make $\sigma$ and $\pi$ as large as possible, while trying to minimize $\omega$. To date, the values $\sigma \approx 45 \mathrm{~cm}^{3} / \mathrm{g}$ and $\pi \approx 8 \mathrm{~cm}^{3} /$ kernel, produced by both an air popper and the microwave, are the largest expansion volume and flake size achieved by industry. These numbers are very high and can only be observed under ideal experimental conditions. The average values achieved by the everyday consumer are $\sigma \approx 36-40 \mathrm{~cm}^{3} / \mathrm{g}$ and $\pi \approx 5-7$ $\mathrm{cm}^{3} /$ kernel [1]. Furthermore, the lowest observed percentage of unpopped kernels is $\omega \approx 6.8 \%$. Once again this is observed under ideal laboratory conditions, with the consumer finding $\omega \approx 10-12 \%$. [1] Obtaining limits better than those reported by industry and food scientists by using a more efficient method of popping popcorn would be of great interest to popcorn producers. There are many other properties of popcorn that are of interest to industry such as sphericity, pericarp or shell thickness, internal temperature, and moisture content. However, an analysis of these characteristics would be mathematically complex, and not as practical for increasing popcorn production $[3,4]$. The purpose of this chapter is to analyze the physics of popcorn production, using some fairly simple thermodynamic principles, and to predict a method for controlling or increasing the size of the popped popcorn. We then compare the results of the theory with various experiments. Then we discuss our results and their application to the popcorn industry.

\section{Thermodynamics of Popping Popcorn}

Before discussing an increase in the size of a popped kernel, we must examine the general physics behind the production of popcorn. A popcorn kernel consists of starch surrounded by a hard exterior shell called the pericarp. Inside every unpopped kernel of popcorn, there is moisture trapped among the starch. When moisture is heated, it is prone to expand. As the popcorn is heated, the starch inside develops a jelly-like consistency, allowing the moisture to uniformly mix with the starch. When the temperature of the heated kernel exceeds the boiling point of water, the moisture inside changes to a gas, causing a sudden expansion in volume. However, the hard shell or pericarp, which coats the outside of the kernel, keeps the gas trapped inside the shell. Hence, the internal pressure of the kernel rises as the popcorn continues to be heated. When the internal pressure of the kernel reaches the yield or breaking point of the shell, the pericarp splits, and the water vapor inside rapidly expands, taking the jelly-like starch with it. The rapid expansion into the surrounding air cools the starch, forming the fluffy substance known as popcorn. This expansion can be modeled as adiabatic because the speed of the expansion does not allow the gas to exchange heat and reach an equilibrium with the environment. Rather, the expansion stops when the pressure of the gas reaches the pressure of the air surrounding the kernel. This is why freshly popped popcorn is still hot. For more details on the popping mechanism, we refer the reader to $[5,6]$. 
The goal of this paper is to discuss how to control the size of the popped kernel of corn. In a recent paper by Hong and Both [7], a method for controlling the size was developed based on an adiabatic model of the popping mechanism. Because the expansion stops when the vapor pressure becomes the same as the surrounding air pressure, all that is needed to examine the change in size is the governing equations immediately after the pericarp has broken. Therefore, the problem of controlling the size reduces to that of interfacial instability and pattern formation. In other words, we are simply dealing with an interface advancing into a chamber. We will now summarize the theory developed in [7].

The dynamic variable in the popcorn system will be the pressure $P$ inside the kernel at some time $t$. Because the popping mechanism is modeled as an adiabatic expansion, the pressure should obey the following law:

$$
P(t) V(t)^{\gamma}=C_{0}=\text { constant, }
$$

where $V(t)$ is the volume of the kernel at $t$ and $\gamma$ is the usual ratio of the specific heats at a constant pressure and a constant volume, $\gamma=C_{p} / C_{v}$. Let $P_{Y}$ be the yield pressure at which the adiabatic expansion begins, and $V_{0}$ be the initial unpopped volume of the kernel. For water vapor, the value of $\gamma$ is known to be $1.3[8]$. Substituting these values into the adiabatic equation, we find the following expression:

$$
P_{Y} V_{0}^{\gamma}=C_{0}=\text { constant, }
$$

where $C_{0}$ can be determined by the yield pressure and the initial volume. For popcorn, we would use a value of $\gamma=1.3$, the accepted value for water vapor [8]. In treating the adiabatic expansion as an interfacial instability, the next step is to perform a linear stability analysis to determine whether the interface is stable against infinitesimal perturbations. The stability analysis was carried out by Both and Hong, where they found the interface was indeed stable. For more details on the stability analysis, see [7].

Knowing that the interface is stable, we are now ready to determine the size of the popped kernel when its internal pressure has reached that of the surroundings, $P_{0}$. If the kernel stops expanding at a set time $t_{f}$, then the internal pressure of the kernel, $P\left(t_{f}\right)=P_{0}$. From this pressure, we can use the adiabatic equation to determine the final volume of the popped kernel at time $t_{f}$,

$$
V\left(t_{f}\right)={\frac{C_{0}}{P_{0}}}^{1 / \gamma}
$$

Recall from Eq.(1) that we have the constant $C_{0}$ in terms of the yield pressure $P_{Y}$ and the initial volume $V_{0}$. Substituting the value of $C_{0}$, we get a useful expression for the final volume,

$$
V\left(t_{f}\right)=V_{0}\left(\frac{P_{Y}}{P_{0}}\right)^{\frac{1}{\gamma}} .
$$

Eq.(3) allows us to control final size of the popped kernel simply by modifying the pressure surrounding the kernel, $P_{0}$. From this equation we can estimate the 
yield pressure, $P_{Y}$ as well. We will use an assumption that kernels of popcorn, both popped and unpopped, are approximately spherical. Now in reality, both the popped and unpopped kernel are far from spherical. However, this spherical assumption is reasonable since we are only interested in creating a simple theory to predict the ability to control the size, as opposed to a complex theory giving the exact change in size for an odd shaped kernel. For a typical kernel of popcorn, popped under normal atmospheric conditions, we estimate that the radius of the kernel increases by a factor of 4 or more. Thus, the increase in the volume of a popped kernel is about a factor of 60 , or $V_{f} / V_{0} \approx 60$. Now we can use Eq.(1) and Eq.(2) to get the following approximation for the ratio of the yield pressure to the pressure of the surroundings:

$$
\frac{P_{Y}}{P_{0}}=\left(\frac{V_{f}}{V_{0}}\right)^{\gamma} \approx 60^{1.3} \approx 200 .
$$

This allows us to estimate the yield pressure as

$$
P_{Y} \approx 200 P_{0},
$$

which is about 200 times greater than atmospheric pressure. As noted in [7], the yield strength of the pericarp at its rupture point is about the same or greater than polyethylene (LDPE) at room temperature [9]. Now using, the equations above, we want to derive an expression allowing the size of the popped kernel to be controlled by adjusting a simple parameter of the system. We first define the variable $\Gamma$ to be the ratio of the final volume $V_{f}$ to the initial volume $V_{0}$,

$$
\Gamma\left(P_{0}\right)=\frac{V\left(t_{f}\right)}{V_{0}},
$$

which we will call the volume expansion rate. Using Eq.(2) and Eq.(4), we get the following expression for $\Gamma$ :

$$
\Gamma\left(P_{0}\right)=\left(\frac{1}{V_{0}}\right)\left(\frac{C_{0}}{P_{0}}\right)^{\frac{1}{\gamma}} .
$$

From Eq.(5), it is obvious that $P_{0}$, the surrounding pressure, is the parameter that will control the increase in size between a kernel's initial and final states. According to this simple thermodynamic theory, the lower the pressure that surrounds the kernel, the larger the increase in volume of the kernel when it pops. Hong and Both [7] took the adiabatic analysis even further and predicted how much the volume of a popped kernel would change with a fixed deviation from atmospheric pressure. In this chapter, we are more interested in testing the qualitative prediction, that one can increase the size of a piece of popcorn by lowering the surrounding pressure, $P_{0}$. Therefore, we will not discuss the details of the quantitative analysis, but instead refer the reader to [7]. Now we present the experiment, the results, and the comparison with the theory. 


\section{The Popcorn Experiment}

There are many ways that one can choose to pop popcorn. The conventional way is to pop it is on the stove in a pan with some oil. Other popular methods of popping include using the microwave oven and the air popper. In choosing our method of popping to test the theory, it was very important to think about the moisture content of the corn and how the heat is distributed to the kernels. According to the literature, moisture content and the distribution of heat among the kernels play a very important role in popping [3,4,6]. Popcorn pops best when its moisture content is somewhere between $11 \%$ and $14 \%$ percent. If the kernels are dry, the internal pressure does not get high enough to break through the pericarp. For kernels with too much moisture, the pericarp becomes soft and cracks prematurely, before the starch has reached its jelly-like state. Therefore it is best to pick a popping method that works best for a wide range of moisture levels. The same is true for heating. If the kernel is not heated evenly, the results will be a burnt unpopped kernel, or a semi-popped kernel that is burnt on one side. It is also important to heat the kernels rapidly, so as to "shock" the kernels into an adiabatic expansion, not giving them time to adjust or equilibrate to the temperature of the surroundings. If the kernels are cooked gradually, starting at a low heat, the pericarp will once again soften and crack, allowing the moisture to escape out slowly rather than popping out rapidly. Since the moisture content of most brands of popcorn is not readily available, we arbitrarily chose to use Jolly-Time popcorn for all of our experiments. We chose the following two methods of popping to test the predictions of the theory: popping with oil in a pan and dry popping in a mesh bottomed pot. In the future, designing an apparatus that uses microwaves to heat the kernels would be another useful way of testing this theory. Not only is the microwave oven the best way to evenly heat your kernels, it is the most efficient and successful method used by the industry to produce popcorn. Direct comparisons could then be made between the experimental results and those reported by popcorn manufaturers. However, in this particular paper, we were only interested in determining whether this thermodynamic model is qualitatively correct in predicting experimental results. For the purposes of this paper, the two chosen methods of heating the kernels were sufficient.

Our experimental method of popping the popcorn in a pot is one that is familiar to industry as well as people in the common household. Many people who make popcorn at home pop it in a pan with some oil on the bottom. We designed an apparatus, shown in Fig.(1), to see if lowering the surrounding pressure in the pot would yield popped kernels with an increased volume. We started with a standard pressure cooker and altered it to include a vacuum pump used to remove the air and hence, lower the pressure in the pot. A thermocouple connected to a thermometer and a pressure gauge were included to allow measurements of the temperature and pressure inside the pot. The temperature of the pot was controlled with a heating pad attached to the bottom of the pot. A shut-off valve was built into the apparatus, allowing us to break the vacuum seal once the experiment was completed. The procedure for conducting 

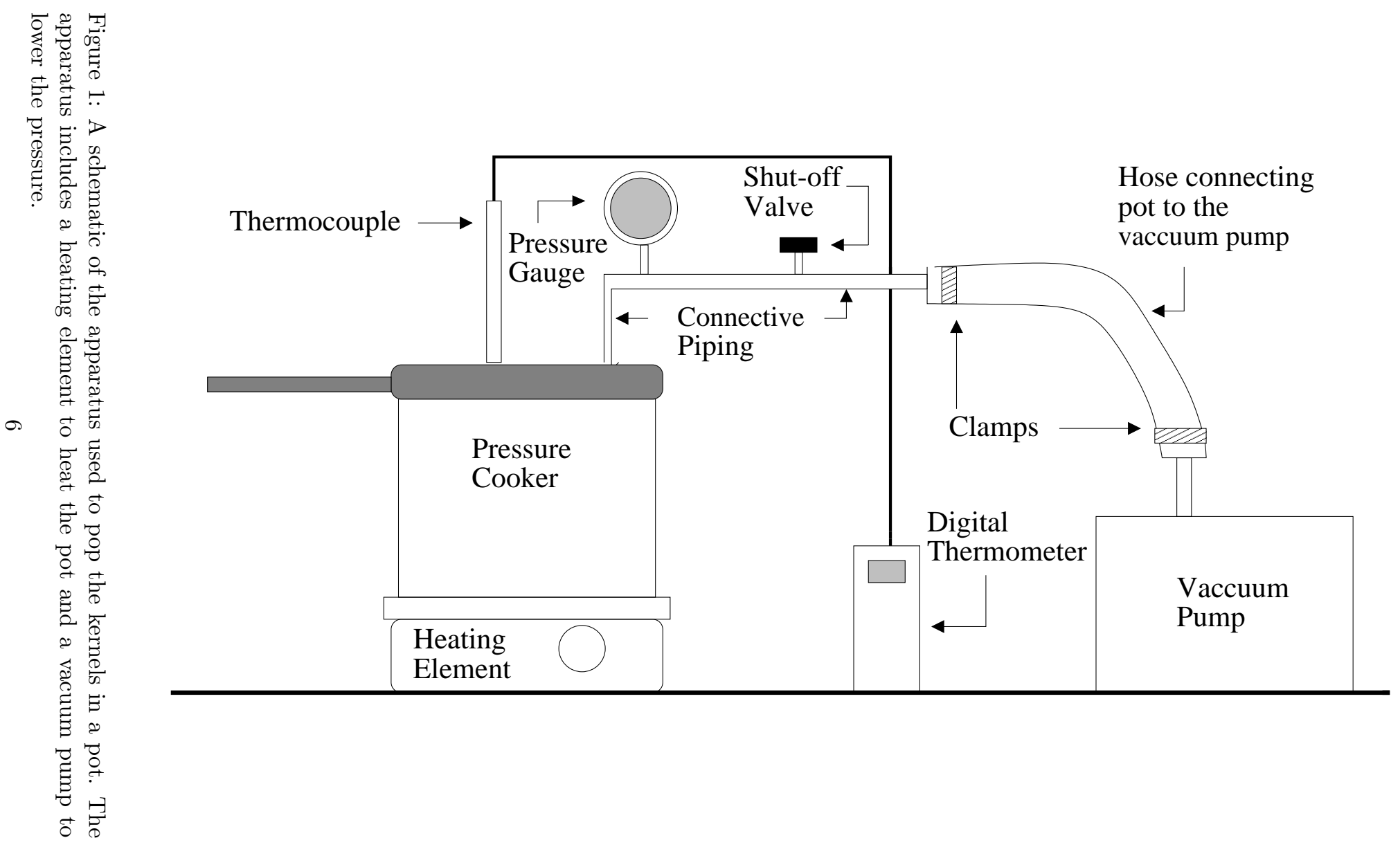
the experiment was fairly simple and very systematic. The first set of data was gathered for popcorn popped in the bottom of the pot with oil. Since most of the experiments conducted in the literature [3,4] worked with $20 \mathrm{~g}$ samples of unpopped kernels, we decided to use the same sample size for our experiments. This sample size resulted in about 135-155 kernels, depending on their size and shape. All samples were kept in sealed bags before use, to prevent them from drying out. The first step was to heat the empty pot to a temperature of $150^{\circ}$ Celsius. Meanwhile, the kernels were mixed with the oil while the pot heated up. When the proper temperature was reached, the oil soaked kernels were put inside the pot, which was then sealed shut. Two runs were done for each set of data, one at atmospheric pressure and one at a pressure reduced to $P \approx 1 / 30$ atm. For the runs conducted at atmospheric pressure, the kernels were left in the sealed pot until the popping was completed. To conduct a run at low pressure, the vacuum pump had to be turned on after the pot was sealed shut with the kernels inside. When the kernels popped, water vapor is released into the pot, causing a slight increase in pressure. Therefore, it was necessary to keep the vacuum pump running throughout the completion of the popping in order to remove this excess water vapor and maintain a low pressure. Once two runs were completed, a comparison could be made between the two samples to see if the volume of the popped kernels had increased under low pressure.

The second method of popping we chose was dry popping the kernels on a steel mesh placed in the bottom of the pot. Oil is used for making popcorn because it coats the kernels and allows for an even distribution of heat over the entire pericarp, or shell. However, we were curious to see if the coating of oil that surrounded each kernel was limiting the size increase of the popcorn as it expanded. Therefore, we chose to eliminate the oil and pop it dry. The mesh was needed to ensure that the heat was more evenly distributed and to keep the kernels away from the bottom of the pan, where they would burn. The procedure was conducted the same as the oil popping method, the only difference being the placing of dry kernels on the mesh, as opposed to the oil soaked mixture used previously.

\section{Experimental Results}

Numerous runs were conducted for both methods of popping. A sample of some results and comparisons for both methods of popping, are shown in Figs.(2-4) as well as Tables 1 and 2. These tables are a small sample of results gathered from individual trials of each experiment. The results in Figs.(2-4) are the average taken over the numerous set of trials we conducted. We made comparisons for the three parameters $\sigma, \pi$, and $\omega$ to see if they were altered by popping at a lower pressure. The first graph, Fig.(2), shows that lowering the pressure more than doubles the value of $\sigma$, the expansion volume. This was the case, regardless of whether we used oil or not. In Fig.(3), one can see that lowering the pressure increased the flake size, $\pi$, by approximately a factor of two as well. At first glance, these results can be deceiving. One might be led to believe that 


\begin{tabular}{|c|c|c|c|}
\hline \multicolumn{4}{|c|}{ Popped in Oil under Atmospheric Pressure } \\
\hline Trial & $\begin{array}{c}\text { Flake Size } \\
\pi\left(\mathrm{cm}^{3}\right)\end{array}$ & $\begin{array}{c}\text { Expansion Volume } \\
\sigma\left(\mathrm{cm}^{3} / \mathrm{g}\right)\end{array}$ & $\begin{array}{c}\text { Unpopped Kernels } \\
\omega(\%)\end{array}$ \\
\hline 1 & 2.76 & 17.5 & 15.3 \\
2 & 2.41 & 10.0 & 40.7 \\
3 & 1.99 & 11.3 & 23.1 \\
4 & 2.35 & 10.0 & 41.8 \\
5 & 2.48 & 12.5 & 29.9 \\
\hline
\end{tabular}

\begin{tabular}{|c|c|c|c|}
\hline \multicolumn{4}{|c|}{ Popped in Oil Under Reduced Pressure } \\
\hline Trial & $\begin{array}{c}\text { Flake Size } \\
\pi\left(\mathrm{cm}^{3}\right)\end{array}$ & $\begin{array}{c}\text { Expansion Volume } \\
\sigma\left(\mathrm{cm}^{3} / \mathrm{g}\right)\end{array}$ & $\begin{array}{c}\text { Unpopped Kernels } \\
\omega(\%)\end{array}$ \\
\hline 1 & 3.75 & 27.5 & 3.4 \\
2 & 3.85 & 27.5 & 4.0 \\
3 & 3.62 & 25.0 & 5.5 \\
4 & 3.79 & 25.0 & 8.3 \\
5 & 4.14 & 27.5 & 3.8 \\
\hline
\end{tabular}

Table 1: A sample of average values per trial of $\pi, \sigma$ and $\omega$ for popcorn popped in oil. The trials on top were conducted at regular pressure, while those on the bottom were done at a reduced pressure of $P \approx 1 / 30 \mathrm{~atm}$.

\begin{tabular}{|c|c|c|c|}
\hline \multicolumn{4}{|c|}{ Popped on Mesh under Atmospheric Pressure } \\
\hline Trial & $\begin{array}{c}\text { Flake Size } \\
\pi\left(\mathrm{cm}^{3}\right)\end{array}$ & $\begin{array}{c}\text { Expansion Volume } \\
\sigma\left(\mathrm{cm}^{3} / \mathrm{g}\right)\end{array}$ & $\begin{array}{c}\text { Unpopped Kernels } \\
\omega(\%)\end{array}$ \\
\hline 1 & 2.34 & 12.5 & 25.7 \\
2 & 2.86 & 15.0 & 27.1 \\
3 & 2.15 & 10.0 & 38.2 \\
4 & 2.17 & 12.5 & 24.8 \\
5 & 2.35 & 12.5 & 27.9 \\
\hline
\end{tabular}

\begin{tabular}{|c|c|c|c|}
\hline \multicolumn{4}{|c|}{ Popped on Mesh under Reduced Pressure } \\
\hline Trial & $\begin{array}{c}\text { Flake Size } \\
\pi\left(\mathrm{cm}^{3}\right)\end{array}$ & $\begin{array}{c}\text { Expansion Volume } \\
\sigma\left(\mathrm{cm}^{3} / \mathrm{g}\right)\end{array}$ & $\begin{array}{c}\text { Unpopped Kernels } \\
\omega(\%)\end{array}$ \\
\hline 1 & 3.96 & 27.5 & 3.5 \\
2 & 4.10 & 27.5 & 6.3 \\
3 & 3.90 & 27.5 & 2.1 \\
4 & 4.01 & 27.5 & 5.8 \\
5 & 3.23 & 22.5 & 8.5 \\
\hline
\end{tabular}

Table 2: A sample of average values per trial of $\pi, \sigma$ and $\omega$ for corn dry popped on a mesh. The trials on top were conducted at regular pressure, while those on the bottom were done at a reduced pressure of $P \approx 1 / 30 \mathrm{~atm}$. 


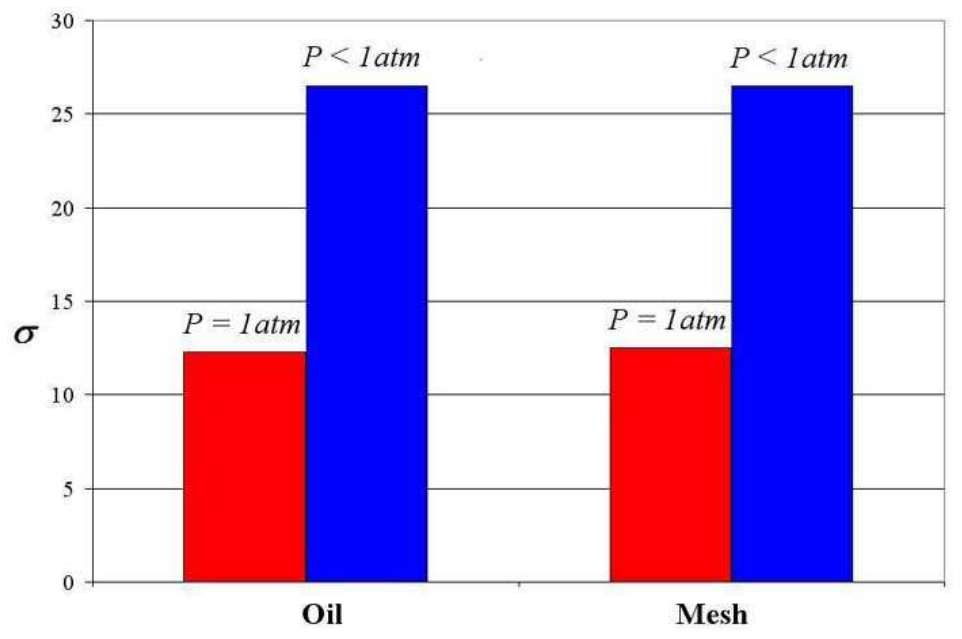

Figure 2: A comparison of $\sigma$ between kernels popped at regular atmospheric pressure and those popped at a reduced pressure of $P \approx 1 / 30 \mathrm{~atm}$. This graph shows the comparison of $\sigma$ for both the oil and mesh popping. 


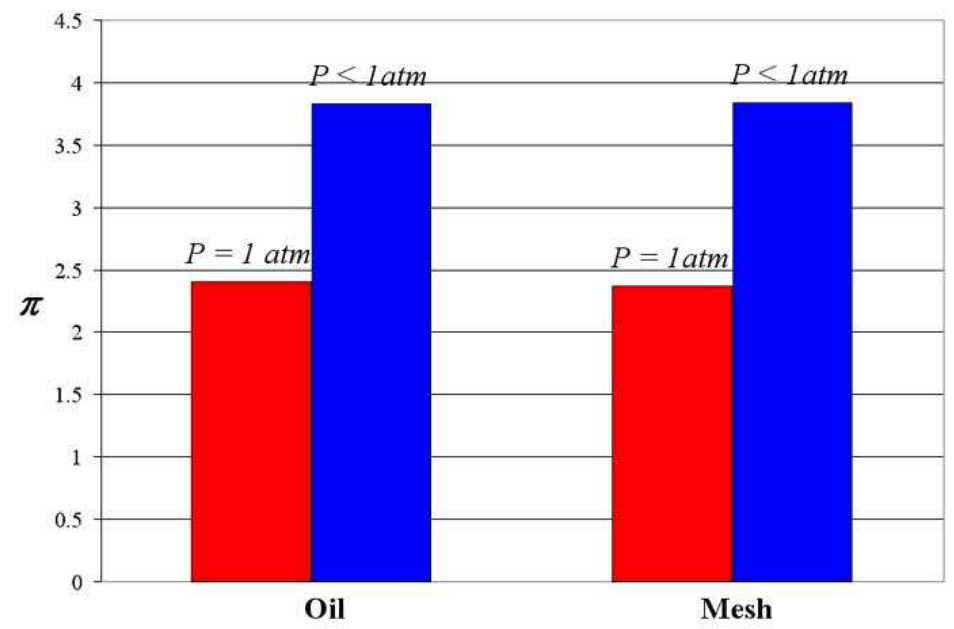

Figure 3: A comparison of $\pi$ between kernels popped at regular atmospheric pressure and those popped at a reduced pressure of $P \approx 1 / 30 \mathrm{~atm}$. This graph shows the comparison of $\pi$ for both the oil and mesh popping. 


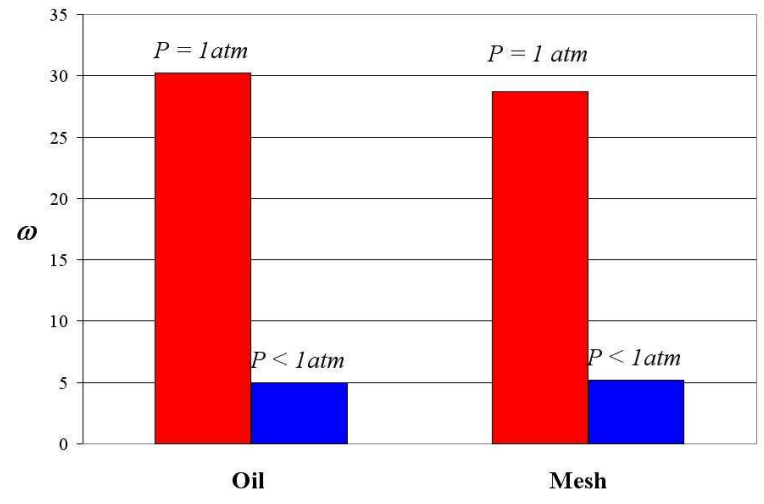

Figure 4: A comparison of unpopped kernels, $\omega$, between kernels popped at regular atmospheric pressure and those popped at a reduced pressure of $P \approx$ $1 / 30 \mathrm{~atm}$. The results are shown for both the runs using oil and the mesh. 
since the volume of the popcorn was approximately doubled, we have produced a popped kernel that looks twice as big. Recall, though, that our calculations were done using a spherical approximation for the volume of a kernel of popcorn. Using the definition for the volume of a sphere, $V=\frac{4}{3} \pi r^{3}$, one can see that if the volume is doubled, the radius is only increased by a factor of $2^{\frac{1}{3}} \approx 1.2$. This is an increase in the radius, but not one that can be observed with the naked eye, specifically, in a popcorn sample containing a variety of shapes and sizes. However, the volume has significantly increased, which can be seen clearly from our results. Under regular popping, $20 \mathrm{~g}$ of unpopped kernels yields one $8 \mathrm{oz}$ cup of popcorn, while $20 \mathrm{~g}$ popped in a low pressure environment yields two 8 oz cups. The predicted increase in size by the theory was qualitatively confirmed by our experiments. Another interesting result that we observed was an extreme decrease in the value of $\omega$, the percentage of unpopped kernels in a sample. As shown in Fig.(4), the amount of wasted kernels decreases by about a factor of 5. This means that popping under a reduced pressure is, in fact, a more efficient method of producing popcorn. This is a result that could be very useful for the popcorn producing industry. Means of production could be made more efficient, reducing the cost acquired by the producers and hence, increasing overall profits.

\section{Conclusion}

It is clear from the presented results that the qualitative predictions of [7] are correct. The popping process in a pot, whether using oil or dry popping with the mesh on the bottom, displayed an increase in the size of the kernels due to the reduced pressure of the surroundings. Popping under reduced pressure also seemed to be a more efficient method of making popcorn, shown by the decrease in unpopped kernels that were observed. The results seem to indicate that regardless of the popping method, it may be possible, on average, to double the volume of popped kernels. Recall that the maximum reported values for the volume expansion, $\sigma$ and the flake size, $\pi$, are reported to be approximately $36-40 \mathrm{~cm}^{3} / \mathrm{g}$ and $5-7 \mathrm{~cm}^{3} /$ kernel, respectively [1,2]. It is important to note that even though we have demonstrated an increase in $\sigma$ and $\pi$ that occurs when the pressure is reduce, our maximum values of $27.5 \mathrm{~cm}^{3} / \mathrm{g}$ and $4.01 \mathrm{~cm}^{3} / \mathrm{kernel}$ are still smaller then those previously reported $[1,2]$. However, those previously reported values were obtained without lowering the pressure, in an air popper or a microwave. So if the pattern observed in our data was to remain true, an air popper or a microwave oven placed in a reduced pressure environment, could possibly produce double the values of the maximums currently observed in the literature today. I would expect at least some increase in the size due to the lowering of the pressure. It is important to keep in mind that the expansion of the starch in the popped kernel does have a physical limit. Doubling the results obtained by the popcorn industry may be well beyond the physical size

limitiations of the start. However, it would be interesting to see if results that are better than those obtained by industrial methods can be produced with a 
more efficient apparatus, such as the microwave oven.

For this idea to catch the interest of popcorn producers, an attempt must be made to obtain results that outdo the maximum values reported by industry. This is a design problem that can be worked out by an engineer and then used to further test this theory. We have clearly shown that the size can be controlled by changing the surrounding pressure. Now it is just a matter of perfecting the mechanism that uses this premise. It is also important to note, that reducing the pressure lowered the unpopped number of kernels by a factor of 5 . This should be of real interest to the popcorn producers. By reducing the number of wasted kernels, manufacturers will get more popcorn for the same cost of supplies. This could be a very effective way to cut down on costs, and hence, make more profit. More work needs to be done to see if the effects produced by lowering the pressure, can be magnified even further. However, we have demonstrated beyond a doubt, that the surrounding pressure is a very important parameter in the production of popcorn.

\section{Acknowledgements}

I would like to acknowledge Dr. Daniel C. Hong for all his work on this project. Without his ingenuity and creative thinking, this project would have never come to fruition. Unfortunately Dr. Hong passed away in 2002 and was unable to see this process to completion. Dr. Hong was a great scientist, colleague, mentor, and friend. The scientific world has lost a wonderful scientist and a fantastic theoretical mind. His presence in the physics community will surely be missed. I would also like to thank Joe Zelinski at Lehigh University for his help in designing and constructing the apparatus used for this experiment. I would also like to acknowledge the physics department at Lehigh University for providing the funds necessary to construct the apparatus.

[1] A. A. Mohamed, R. B. Ashman, and A. W. Kirleis, Journal of Food Science, 58, No.2, 342(1993).

[2] R. C. Hoseny, K. Zeleznak, and A. Abdelrahman, Journal of Cereal Science, 1, 43(1983).

[3] T. H. Roshdy, K. Harakawa, and H. Daun, Journal of Food Science, 49, 1412(1984).

[4] C. G. Haugh, R. M. Lien, R. E. Hanes, and R. B. Ashman, Trans. Am. Soc. Agric. Eng., 19, 168(1976).

[5] Robert G. Hunt, The Physics Teacher, April, 230(1991).

[6] W. J. Silva et al, Nature, April, No. 6419,362(1993).

[7] D. C. Hong and Joseph A. Both, Physica A, 289, 557(2001). 
[8] CRC Handbook of Physics and Chemistry, CRC Press,Boca Raton, Florida,D$172,(1985)$.

[9] N. E. Dowling, Mechanical Behavior of Materials, Prentice Hall, Englewood Cliffs, New Jersey, 158(1993). 